\title{
Electrical Stimulation Pain Therapy
}

National Cancer Institute

\section{Source}

National Cancer Institute. Electrical Stimulation Pain Therapy. NCI Thesaurus. Code

C116535.

The use of external electrical current to stimulate nerves for the purpose of reducing pain. 